University of Nebraska - Lincoln

DigitalCommons@University of Nebraska - Lincoln

Faculty Publications from the Department of Electrical \& Computer Engineering, Department Electrical and Computer Engineering

$9-1-2000$

\title{
Dielectric tensor for interfaces and individual layers in magnetic multilayer structures
}

\author{
Xiang Gao \\ University of Nebraska-Lincoln \\ Michael J. DeVries \\ University of Nebraska-Lincoln \\ Daniel W. Thompson \\ University of Nebraska-Lincoln, dthompson2@unl.edu \\ John A. Woollam \\ University of Nebraska-Lincoln, jwoollam1@unl.edu
}

Follow this and additional works at: https://digitalcommons.unl.edu/electricalengineeringfacpub

Part of the Electrical and Computer Engineering Commons

Gao, Xiang; DeVries, Michael J.; Thompson, Daniel W.; and Woollam, John A., "Dielectric tensor for interfaces and individual layers in magnetic multilayer structures" (2000). Faculty Publications from the Department of Electrical and Computer Engineering. 18.

https://digitalcommons.unl.edu/electricalengineeringfacpub/18

This Article is brought to you for free and open access by the Electrical \& Computer Engineering, Department of at DigitalCommons@University of Nebraska - Lincoln. It has been accepted for inclusion in Faculty Publications from the Department of Electrical and Computer Engineering by an authorized administrator of DigitalCommons@University of Nebraska - Lincoln. 


\title{
Dielectric tensor for interfaces and individual layers in magnetic multilayer structures
}

\author{
Xiang Gao, Michael J. DeVries, Daniel W. Thompson, and John A. Woollam \\ Center for Microelectronic and Optical Materials Research, and Department of Electrical Engineering, \\ University of Nebraska, Lincoln, Nebraska 68588-0511
}

(Received 21 February 2000; accepted for publication 30 May 2000)

\begin{abstract}
The magneto-optical Kerr response of metallic magnetic multilayers has been studied by determining the dielectric tensors (dielectric functions) for individual layers, including the magnetic and nonmagnetic interfacial layers. The diagonal components of these tensors were determined using in situ ellipsometric analysis, where the ellipsometric data were taken in real time during multilayer deposition. The off-diagonal components were determined by regression fitting magneto-optic polar Kerr rotation and ellipticity data to models supported by electromagnetic theory. The Voigt parameters (ratio between off-diagonal and diagonal components of dielectric tensors) were determined from these model fits. Higher magnitudes for the Voigt parameters were found at interfaces, corresponding with stronger Kerr responses observed in those materials. Five different magnetic multilayer systems were studied, including $\mathrm{Pt} / \mathrm{Co}, \mathrm{Pd} / \mathrm{Co}, \mathrm{Au} / \mathrm{Co}, \mathrm{Cu} / \mathrm{Co}$, and $\mathrm{Pt} / \mathrm{Fe}$ multilayer structures. The Voigt parameters for the magnetic layers and magneticnonmagnetic interfaces in all five structures were determined, and in turn the dielectric tensors for the respective layers were also determined. (C) 2000 American Institute of Physics.
\end{abstract} [S0021-8979(00)05317-2]

\section{INTRODUCTION}

Ferromagnetic-transition metal multilayer structures have been studied as magneto-optic (MO) media for more than a decade. ${ }^{1-16}$ Both Co-based and Fe-based magnetic multilayers show good blue responses which are important for higher density magneto-optical recording. ${ }^{4-10}$ Large Kerr responses in the blue were believed to be related to the presence of the transition metal (especially $\mathrm{Pt}$ and $\mathrm{Pd}$ ) in the interfacial region. This can be easily observed by comparing Kerr spectra for $\mathrm{Co} / \mathrm{Pt}$ multilayers to a Kerr spectrum for a single Co layer. ${ }^{7}$ The comparison in Ref. 7 shows that when the Pt layer decreases below a certain thickness, the Kerr response in the blue range is larger for the multilayers than for a single Co layer; however, it is smaller for the multilayers in the red range. Ab initio calculations have been used to calculate the moments of both magnetic atoms and transition metal atoms with the spin-orbit interaction included in the calculations. ${ }^{11-13}$ Calculated results from Ref. 13 show that induced moments for the transition metal atoms at the interface are much larger than for atoms away from the interface. The dielectric tensor was also calculated using ab initio calculations, and the Kerr spectra were in turn determined from the calculated dielectric tensor., ${ }^{9,13}$

In previous experimental work, the dielectric tensors for magnetic multilayer structures have been measured by treating them as single layers. ${ }^{9,14-16}$ The Kerr spectra were also simulated by treating the individual layers separately within the framework of multilayer structures. ${ }^{7,17,18}$ In the simulations in Ref. 7, spin-polarized Pt (or Pd) layers were included between the unpolarized Pt (or Pd) layer and the magnetic Co layer. Tabulated optical constants were used for the diagonal part of the dielectric tensor for the polarized Pt (or
Pd) layers and the Co layers, as well as for the unpolarized Pt (or Pd) layers. The off-diagonal part of the dielectric tensor for the Co layers and the polarized Pt (or Pd) layers were simulated using results from other materials.

In our previous work, the diagonal part of the dielectric tensor for the individual layers in Pt/Co multilayer structures were determined by in situ spectroscopic ellipsometry (SE) analysis. ${ }^{17}$ The off-diagonal parts of both the Co layer and the Co-Pt interface were determined by combining the diagonal results obtained from in situ SE and an ex situ magnetooptic measurements. ${ }^{18,19}$ The results show that the magnitude of the off-diagonal part of the dielectric tensor for the interfaces was significantly larger than for the magnetic layers, leading to the conclusion that the $\mathrm{Pt}-\mathrm{Co}$ interfaces have much larger contributions to the total MO response than do the Co layers in regions away from the interfaces.

In this work, a group of different magnetic/nonmagnetic $(\mathrm{Pt} / \mathrm{Co}, \mathrm{Pd} / \mathrm{Co}, \mathrm{Au} / \mathrm{Co}, \mathrm{Cu} / \mathrm{Co}$, and $\mathrm{Pt} / \mathrm{Fe})$ multilayer structures made by direct current $(\mathrm{dc})$ magnetron sputtering were studied for their optical and magneto-optical properties using the same experimental analysis approach as used in our previous work. Results show that different strengths of interfacial contributions were found for different combinations of magnetic and nonmagnetic materials.

\section{THEORY AND EXPERIMENT}

The measured polar magneto-optic response of a multilayer structure is determined by the pseudo dielectric tensor (the dielectric tensor when the multilayer structure is assumed to be a bulk "substrate"). The pseudo-dielectric tensor is a nonfundamental entity influenced by the thickness of each constituent layer in the multilayer structure, as well 

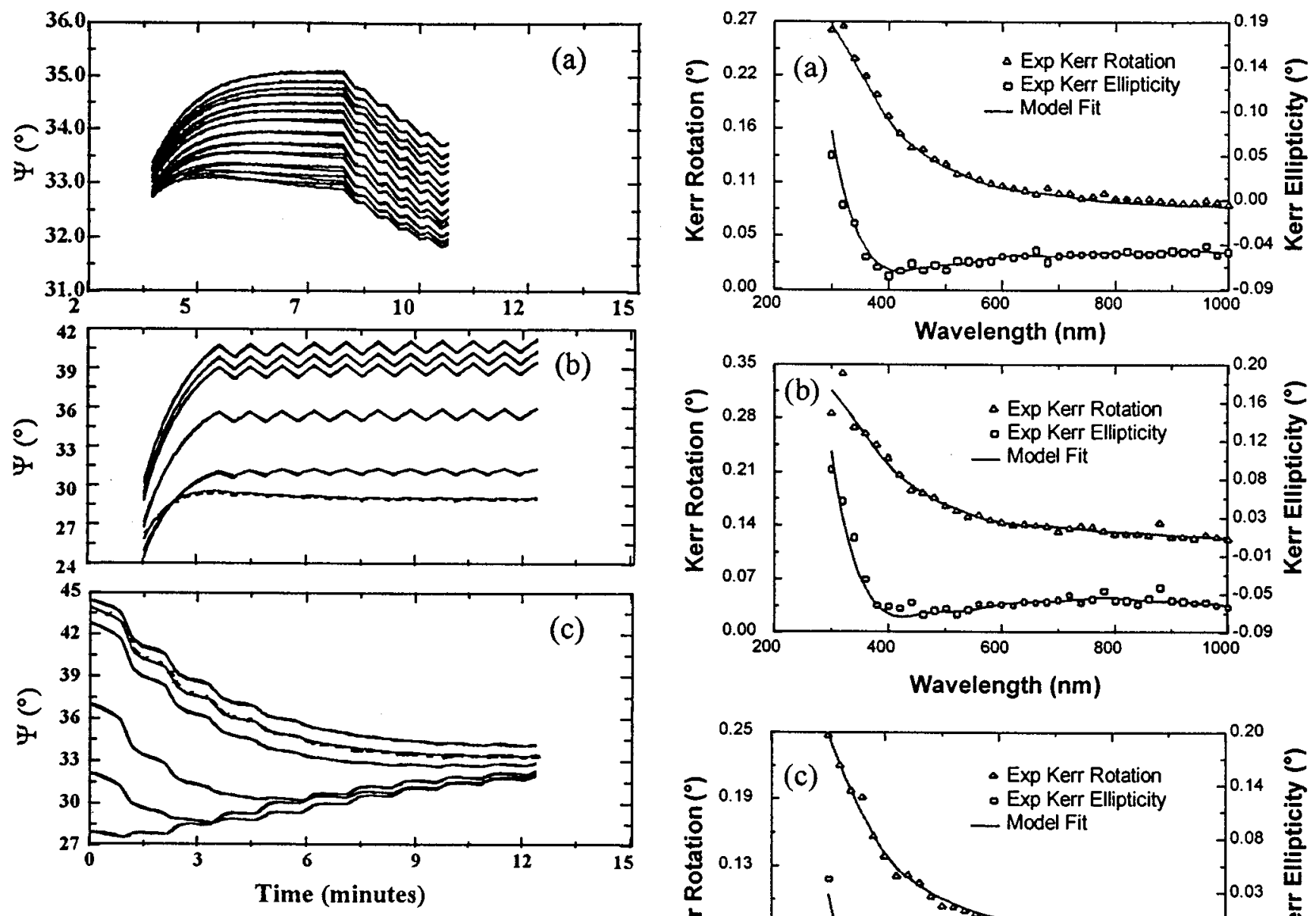
(amplitude of complex reflectance ratio of $p$ - to $s$-polarization states) taken during growth of (a) $\mathrm{Pt} / \mathrm{Co}$, (b) $\mathrm{Au} / \mathrm{Co}$, and (c) $\mathrm{Pd} / \mathrm{Co}$ multilayer structures. The solid curves are results of regression fits. Data were taken at 44 wavelengths simultaneously, but only selected wavelength data (from 415 to 764 $\mathrm{nm}$, as marked) and corresponding regression fits are shown. Note in this and some subsequent figures the experimental data and "fit" data are so close together they are often indistinguishable.

as by the dielectric tensor of each layer. To understand the measured response, one needs to know the contributions from the different layered regions of the structure. For layered MO materials, there are off-diagonal components for the magnetic layers as well as for the interfaces between the magnetic and nonmagnetic layers. The dielectric tensor for each individual layer is given by

$$
\widetilde{\varepsilon}=\left(\begin{array}{ccc}
\widetilde{\varepsilon}_{x x} & \widetilde{\varepsilon}_{x y} & 0 \\
-\widetilde{\varepsilon}_{x y} & \widetilde{\varepsilon}_{x x} & 0 \\
0 & 0 & \widetilde{\varepsilon}_{x x}
\end{array}\right) .
$$

By normalizing this dielectric tensor, we get

$$
\widetilde{\varepsilon}=\widetilde{\varepsilon}_{x x} \cdot\left(\begin{array}{ccc}
1 & -i \widetilde{Q} & 0 \\
i \widetilde{Q} & 1 & 0 \\
0 & 0 & 1
\end{array}\right),
$$

where $\widetilde{Q}=Q_{1}+i Q_{2}$ is the Voigt parameter and $\widetilde{\varepsilon}_{x x}=\varepsilon_{x x 1}$ $+i \varepsilon_{x x 2}$ is the isotropic dielectric function. The spectrally dependent dielectric tensor can be determined by determining $\widetilde{Q}$ and $\widetilde{\varepsilon}_{x x}$ as a function of wavelength.

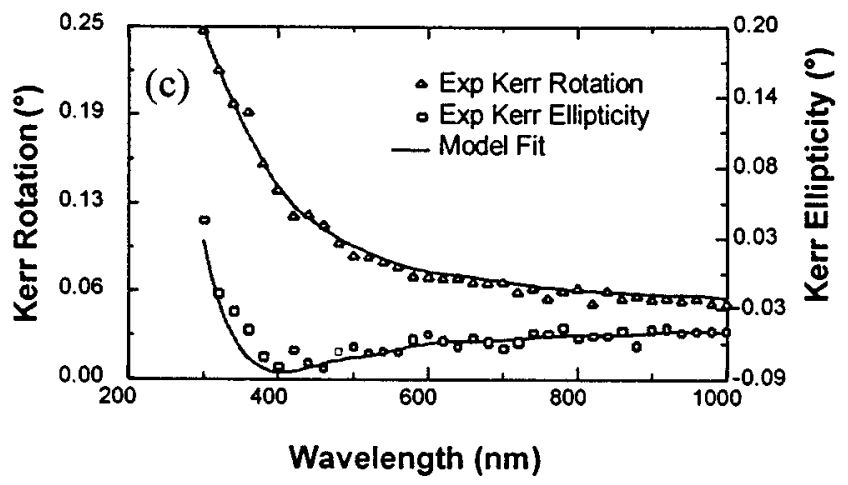

FIG. 2. Magneto-optic data taken from three Pt/Co multilayer structures. Parameters for each sample, as defined in Fig. 4, are: (a) $x=1.15$, $y=0.175, n=20 ;$ (b) $\mathrm{x}=1.15, y=0.55, n=20 ;$ (c) $\mathrm{x}=1.15, y=0.175$, $n=10$.

Samples were de sputter deposited in an Ar environment with an Ar pressure of $10 \mathrm{mTorr}$. The base pressure of the vacuum chamber was $\sim 3 \times 10^{-7}$ Torr. During deposition, in situ spectroscopic ellipsometry data were taken in real time. The thicknesses of the individual layers in the multilayer structures and the diagonal part of the dielectric tensor $\widetilde{\varepsilon}_{x x}$ of each of the different constituent layers were determined simultaneously by after-growth SE analysis of the in situ data. ${ }^{17}$

The polar magneto-optic Kerr responses from these samples were taken ex situ using a modified spectroscopic ellipsometer. ${ }^{18}$ The measured Kerr response is a function of the layer thickness of each individual layer in the multilayer structures, as well as the dielectric tensor of each layer. Since the thickness and the diagonal part of the dielectric tensor $\widetilde{\varepsilon}_{x x}$ have already been determined by in situ SE, the Voigt parameter $\widetilde{Q}$ is the only variable to be determined from model fitting $\widetilde{Q}$ to the MO Kerr rotation and ellipticity data. As both the $\widetilde{\varepsilon}_{x x}$ and $\widetilde{Q}$ are determined, the dielectric tensor 

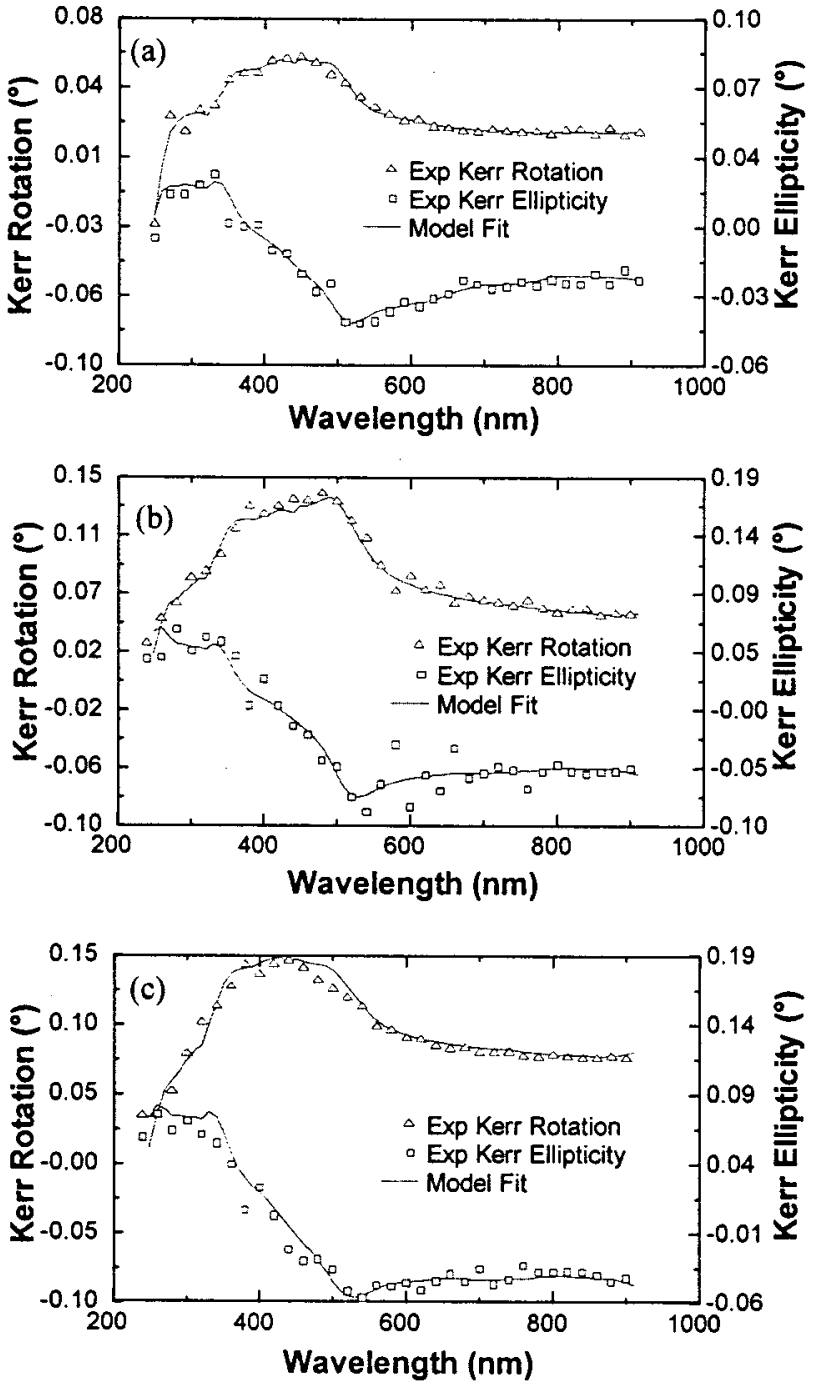

FIG. 3. Magneto-optic data taken from three $\mathrm{Au} / \mathrm{Co}$ multilayer structures. Parameters for each sample are: (a) $\mathrm{x}=1.1, y=0.20, n=20$; (b) $\mathrm{x}=1.1$, $y=0.44, n=10 ;$ (c) $\mathrm{x}=1.1, y=0.44, n=20$.

[Eq. (1)] is obtained for each layer, including interfacial layers.

In this work, the nonmagnetic metal layers in the multilayer systems are treated as isotropic materials $\left(\widetilde{\varepsilon}_{x y}\right.$ equal to zero), and the magnetic layer $\mathrm{Co}$ and $\mathrm{Fe}$ layers are anisotropic with nonzero $\widetilde{\varepsilon}_{x y}$. The interfacial regions between the magnetic layers and the nonmagnetic layers are also treated as magnetic layers with nonzero $\widetilde{\varepsilon}_{x y}$. The isotropic $\widetilde{\varepsilon}_{x x}$ for the interface is simulated by an effective medium approximation by combining the $\widetilde{\varepsilon}_{x x}$ data of the adjacent magnetic and nonmagnetic layers.

\section{RESULTS AND ANALYSIS}

Representative in situ spectroscopic ellipsometry data, taken on $\mathrm{Pt} / \mathrm{Co}, \mathrm{Au} / \mathrm{Co}$, and $\mathrm{Pd} / \mathrm{Co}$ multilayer structures, are shown in Fig. 1. The thicknesses and the diagonal part of the dielectric tensor for each layer were determined by an aftergrowth regression analysis on the in situ SE experimental data. During the regression fits, the thicknesses of both the magnetic and nonmagnetic layers, and the diagonal part of

\begin{tabular}{|llr|}
\hline 4 & Au, Cu, Pt or Pd & $x \mathrm{~nm}$ \\
\hline 3 & Interface & $0.2 \mathrm{~nm}$ \\
\hline 2 & Magnetic layer (Co or Fe rich) & $\mathrm{y} \mathrm{nm}$ \\
\hline 1 & Interface & $0.2 \mathrm{~nm}$ \\
\hline 0 & $\mathrm{Au}, \mathrm{Cu}$, Pt or Pd & $1 \mathrm{~mm}$ \\
\hline
\end{tabular}

FIG. 4. The general model for all fits, where layer 1 and layer 3 are interfaces with the thicknesses and dielectric tensor for both layers coupled during regression. Layer 2 and layer 4 are magnetic and nonmagnetic, respectively.

the dielectric tensor $\left(\widetilde{\varepsilon}_{x x}\right)$ are allowed to float as variables. The regression fits stop when the mean square error (MSE) reaches a minimum. A fit is considered good when the regression fit results in overlap between the fitted data and the experimental data, while the corresponding MSEs are normally very small. The degree of uncertainty of each variable is provided by $90 \%$ confidence limit calculations, as well as the correlation matrices. Low correlation between variables is desired, allowing parameters to be uniquely determined. Starting values of layer thicknesses and diagonal parts of the dielectric tensor were determined by in situ SE diagnostics for single film depositions under identical growth situations for the multilayer growth. These were done in the same vacuum pump down, immediately prior to making multilayers, and done using the same dc power, same Ar pressure, and same underlayer materials $(\mathrm{Pt}$ or $\mathrm{Au})$. The uncertainty in parameter determination can be further tested by changing the starting value of the variables within reasonable ranges for the regression analysis. In the present work, correlation between thickness and $\widetilde{\varepsilon}_{x x}$ of individual layers is significantly reduced due to the sawtooth features seen in Fig. 1, created by the optical contrast between different materials. The sawtooth pattern is due to the repeating structures in different growth stages, which allows multiple-sample regression analysis, and reduces the uncertainty of the parameter fitting results. The $90 \%$ confidence limits of the fits for different physical structures were all acceptably small, within $\pm 3 \%$ of the final values. Different starting values for the thicknesses and $\widetilde{\varepsilon}_{x x}$ values were also used, and the final results were the same. The bilayer thicknesses for the multilayer structures were also confirmed by x-ray diffraction measurements, and agreed with the ellipsometric results to better than one percent. More detailed analysis of the in situ ellipsometric diagnostic procedure for multilayer structures was published elsewhere. ${ }^{17}$

Polar Kerr rotation data were then taken on all samples. Data were acquired by saturating the magnetization in a positive field of $1 \mathrm{~T}$ at the selected wavelength, then reversing the field and averaging the data. The next wavelength was then selected, field reversals done, and so forth. (Occasional full hysteresis loop Kerr data were acquired to verify full saturation of the magnetization.) Representative data as a function of wavelength of light are shown for the Pt/Co and $\mathrm{Au} / \mathrm{Co}$ multilayer structures in Figs. 2 and 3, respectively. 

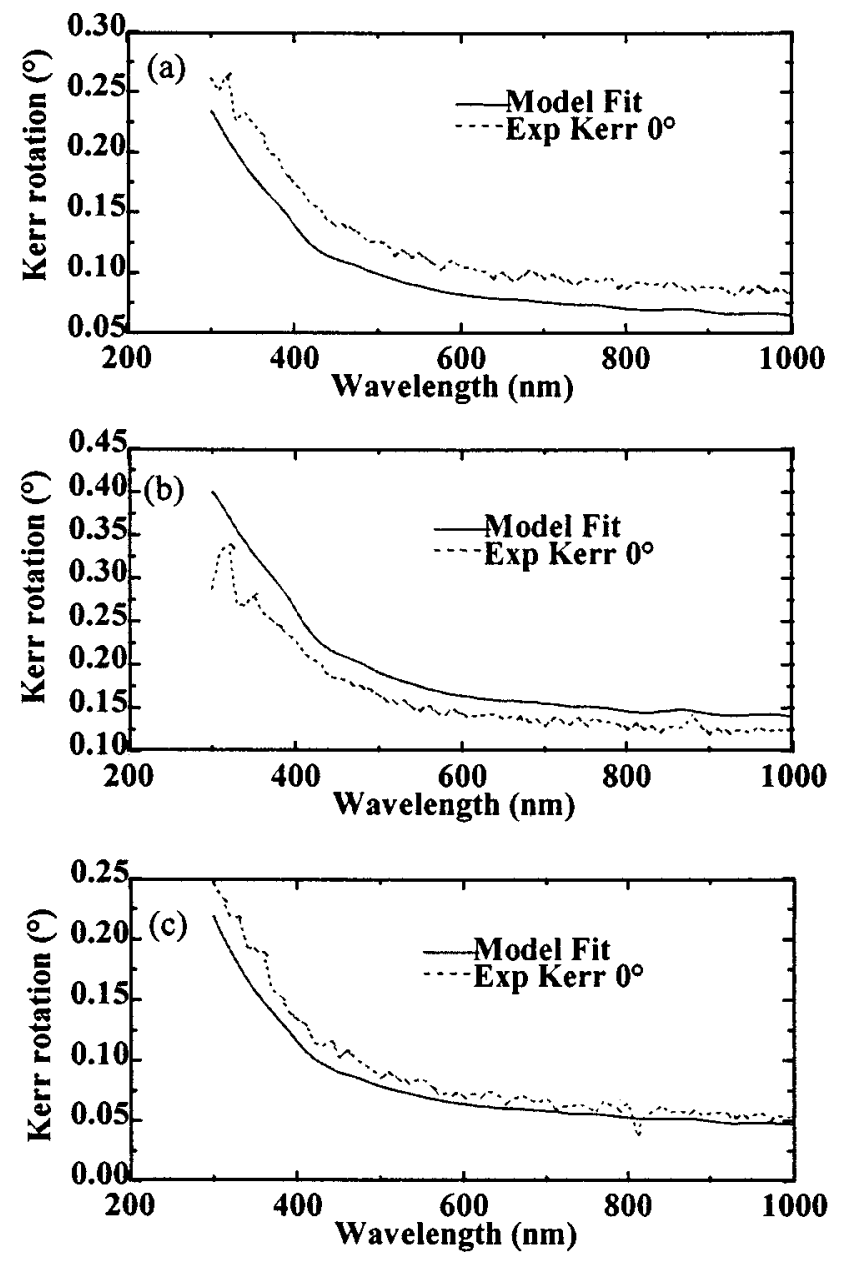

FIG. 5. Regression fits for the samples in Fig. 2 without the addition of the interfaces. The samples in (a)-(c) correspond to the samples in Figs. 2(a)2(c), respectively. The mean square errors (MSE) for fits in this and 6 are about five times larger than the corresponding fits in Figs. 2 and 3 where interfaces are assumed in the model.

Figure 4 shows the models used for regression fits for all structures. The thickness and the diagonal part of the dielectric tensor of each layer were predetermined from the in situ SE analysis, as previously discussed. An actual "interface" layer was not seen in the in situ SE data, suggesting that this interface is magnetic and not structural in nature, or if structural, too small to be seen in the in situ SE data alone. That is, the ability to detect the interfacial magnetic interface is dependent on the multiple sample, multiple data type (SE plus MO) measurements, and analysis to follow.

In order to analyze the different contributions from both the magnetic Co rich (or Fe rich) layers and the interfaces between the magnetic and nonmagnetic layers, the total magnetic portion of each bilayer was divided into two regions: a Co rich (or Fe rich) magnetic region, and an interfacial region. (Introduction of interfacial layers, taking care not to introduce correlated parameters, is a common and successful procedure followed in ellipsometric studies of complex multilayer structures, and is regularly supported by independent measurements by other techniques. ${ }^{20}$ ) Then, the Voigt parameters for both the magnetic and interface regions in the multilayer systems were determined by regression fits. Dur-
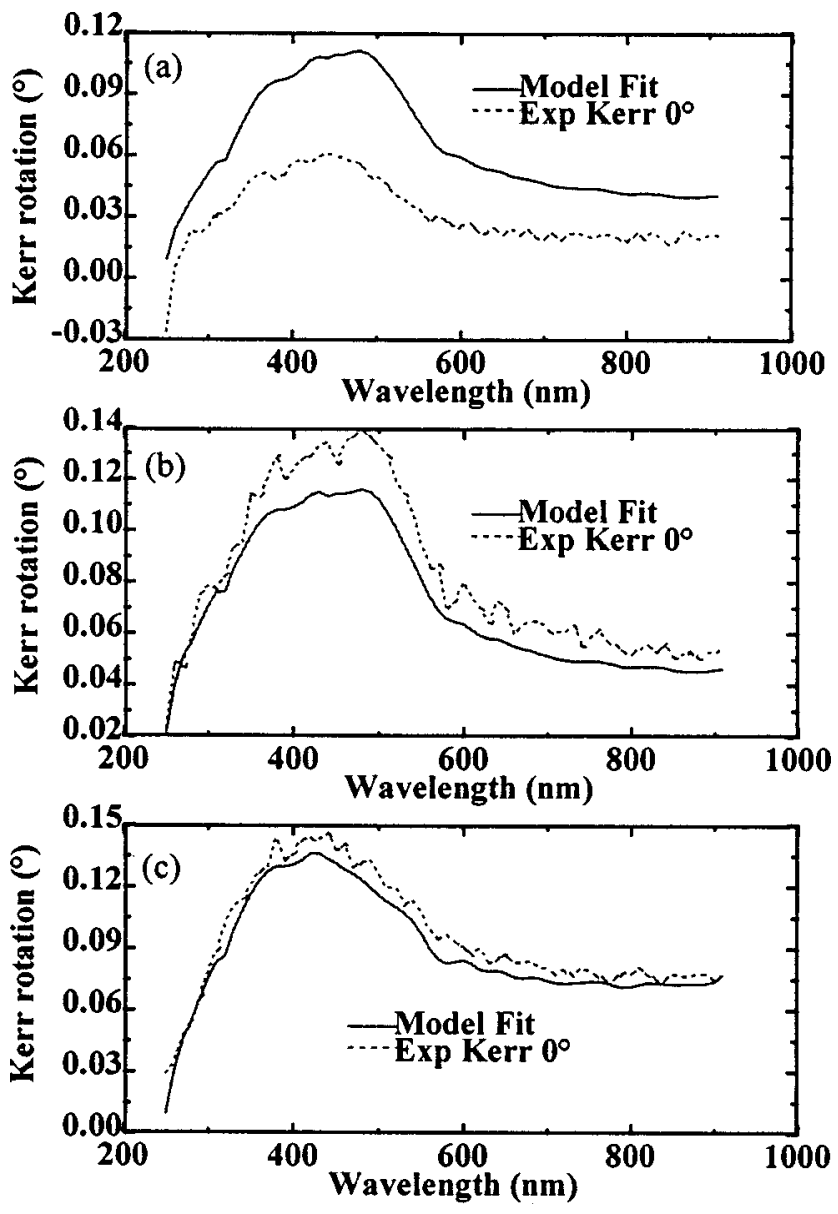

FIG. 6. Regression fits for the samples in Fig. 3 without the addition of the interfaces.

ing the regression fits, multi-sample analysis was also used to obtain noncorrelated results for the Voigt parameters for both the magnetic layers and interfaces. This powerful method allows unique determination of independent Voigt parameters, far exceeding what is possible using data from only a single sample. ${ }^{20}$ Figures 2 and 3 show two groups of fitting results for $\mathrm{Pt} / \mathrm{Co}$ and $\mathrm{Au} / \mathrm{Co}$ structures, respectively, with three different structures in each group. The three $\mathrm{Pt} / \mathrm{Co}$ (or $\mathrm{Au} / \mathrm{Co}$ ) samples have different Co rich layer thicknesses and different repeat numbers of period. During fits for each structure, the Voigt parameters of the Co rich layers were coupled as one variable for all three different samples, and the interfacial Voigt parameters for all the interfaces were also coupled. Thus, there are only two complex variables (That is, four unknowns; two real and two imaginary) for each group at each wavelength. There are three different sets of spectral data from three different samples structures (That is, nine sets of spectral data to determine four sets of spectrally dependent unknowns). As a result, the correlation between the Voigt parameter for the magnetic layers and the interfacial layers was drastically reduced and the measurements are largely independent. Thus, determination of Voigt parameters is unique, within the limits of regression analysis.

Based on $a b$ initio calculations that find very small moments induced on the second nearest atomic neighbor in the Pt layer, ${ }^{13}$ a single atomic layer interface was assumed for all 

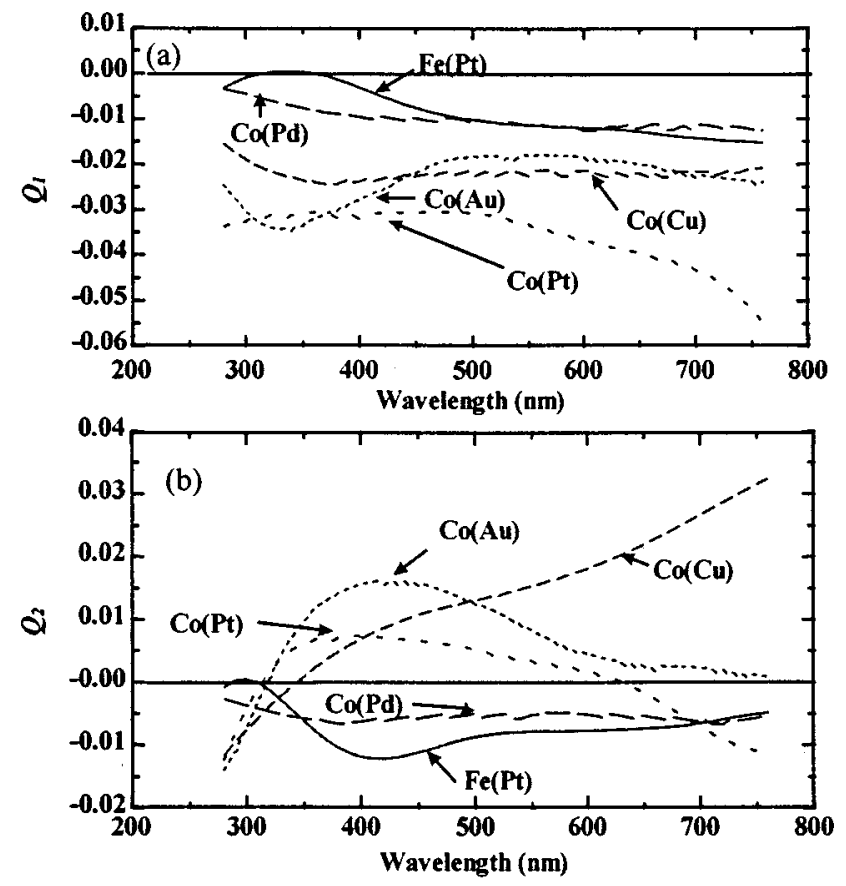

FIG. 7. Results of the magneto-optic analysis: Voigt parameters (a) $Q_{1}$ and (b) $Q_{2}$ for the magnetic layers for each multilayer structure.

the structures in the magneto-optical model used for this regression analysis. The diagonal part of the dielectric tensor of the interfaces is represented by an effective medium approximation, as previously introduced.

The necessity of the addition of the interface layers can be demonstrated by the poor regression fits found when only the Voigt parameters for the magnetic layers were fit to the same sets of data as in Figs. 2 and 3. As shown in Figs. 5 and 6, the fits are much worse than fits in Figs. 2 and 3. That is, removal of the interface as having independent magnetooptic properties definitively worsens the regression fits.

The same regression analysis approach was performed on the MO Kerr data taken from $\mathrm{Cu} / \mathrm{Co}, \mathrm{Pd} / \mathrm{Co}$, and $\mathrm{Pt} / \mathrm{Fe}$ multilayer structures. The results of the Voigt parameters $Q_{1}$ and $Q_{2}$ [real and imaginary parts of $Q$ introduced in Eq. (2)] for the magnetic layers and interfacial regions for all the structures are shown in Figs. 7 and 8, respectively. Indeed, Voigt parameters for those interfaces are significantly different from those for the "magnetic" regions.

From Eq. (2), the off-diagonal components in the dielectric tensor can be determined by,

$$
\widetilde{\varepsilon}_{x y}=\widetilde{\varepsilon}_{x x} \cdot\left(i Q_{1}-Q_{2}\right)
$$

where $Q_{1}$ and $Q_{2}$ are the real and imaginary parts of the Voigt parameter. $\widetilde{\varepsilon}_{x x}$ is the complex diagonal component of the dielectric tensor, which is the same as the diagonal part of the dielectric tensor determined by in situ SE. The amplitudes of peaks in the $\widetilde{\varepsilon}_{x y}$ spectra are related to the strength of the spin-orbit coupling, ${ }^{21}$ and the magnitudes of Kerr rotation and ellipticity are proportional to the absolute value of $\widetilde{\varepsilon}_{x y} \cdot{ }^{22}$ The results for $\widetilde{\varepsilon}_{x y}$ of the magnetic layers and the interface layers for all the multilayer systems are shown in Fig. 9. By comparison, one can see that in $\mathrm{Pt} / \mathrm{Co}$ and $\mathrm{Pt} / \mathrm{Fe}$
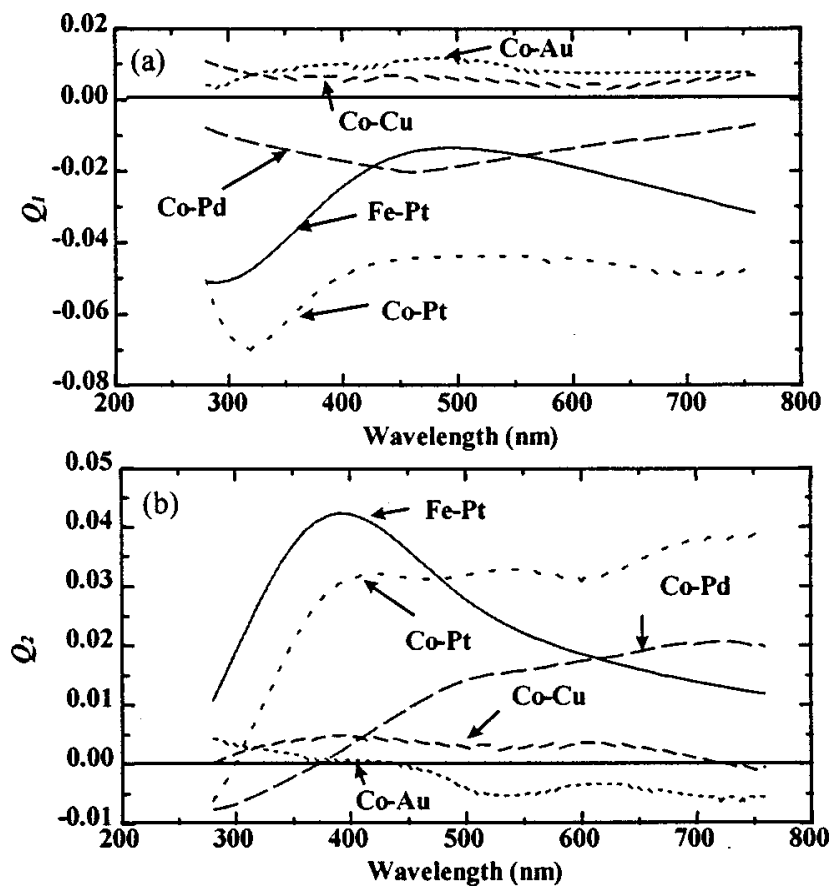

FIG. 8. Voigt parameters (a) $Q_{1}$ and (b) $Q_{2}$ for the interfacial layers for each multilayer structure.

multilayer systems, the interfaces between the magnetic and nonmagnetic layers ( $\mathrm{Pt}-\mathrm{Co}$ and $\mathrm{Pt}-\mathrm{Fe}$ ) have larger contributions to the $\mathrm{MO}$ responses than do the magnetic Co rich and $\mathrm{Fe}$ rich layers. On the contrary, in the $\mathrm{Cu} / \mathrm{Co}$ and $\mathrm{Au} / \mathrm{Co}$ multilayer systems, the $\mathrm{Cu}-\mathrm{Co}$ and $\mathrm{Au}-\mathrm{Co}$ interfaces have smaller contributions than do the magnetic layers. Also seen is that the Pt-Co and Pt-Fe interfaces have the largest absolute values for $\widetilde{\varepsilon}_{x y}$ of all the multilayer systems. However,
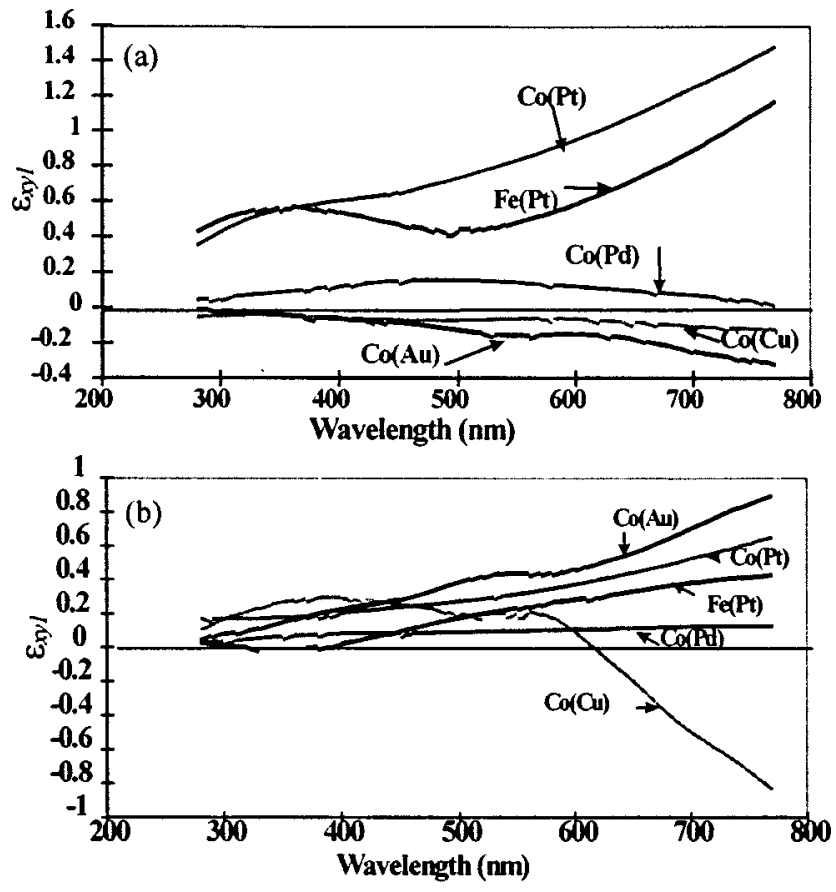

FIG. 9. Real part of the off-diagonal component $\widetilde{\varepsilon}_{x y}$ of the dielectric tensor $\widetilde{\varepsilon}$ calculated from the Voigt parameters for different structures for (a) interfaces and (b) magnetic layers. 

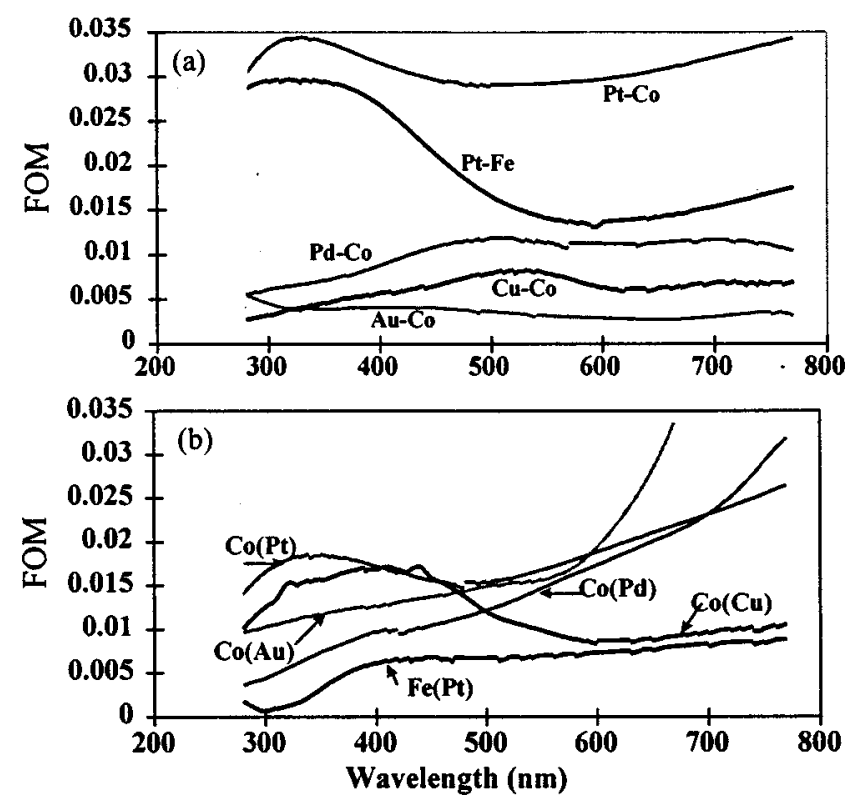

FIG. 10. Intrinsic figures of merit as defined in Eq. (4) for the (a) interfacial layers and (b) magnetic layers for the different structures.

$\widetilde{\varepsilon}_{x y}$ for the Fe rich layer in the Fe/Co structure is smaller than in the Co-based structures, which leads to overall better responses from $\mathrm{Pt} / \mathrm{Co}$ structures than from $\mathrm{Pt} / \mathrm{Fe}$ structures.

The intrinsic figures of merit (FOM) for all interfaces and magnetic layers are calculated (as discussed by Fu et al.) using. ${ }^{23}$

$$
\mathrm{FOM}=\frac{\left|\widetilde{\varepsilon}_{x y}\right|}{2 \operatorname{Im}\left(\widetilde{\varepsilon}_{x x}\right)} .
$$

Results are shown in Fig. 10. We also see that the highest FOMs at short wavelengths are from the $\mathrm{Pt}-\mathrm{Co}$ and $\mathrm{Pt}-\mathrm{Fe}$ interfaces.

\section{SUMMARY AND CONCLUSIONS}

In this work, a group of different magnetic/nonmagnetic $(\mathrm{Pt} / \mathrm{Co}, \mathrm{Pd} / \mathrm{Co}, \mathrm{Au} / \mathrm{Co}, \mathrm{Cu} / \mathrm{Co}$, and $\mathrm{Pt} / \mathrm{Fe}$ ) multilayer structures made by dc magnetron sputtering were studied for their optical and magneto-optical properties. The combination of in situ SE and ex situ multi-sample ellipsometric analysis is a powerful method to determine MO dielectric properties for multilayer magnetic structures, and was used to determine the optical parameters of these multilayers.

Results show that different strengths of interfacial contributions were found for different combinations of magnetic and nonmagnetic materials. The off-diagonal $\widetilde{\varepsilon}_{x y}$ of the dielectric tensor is larger for $\mathrm{Pt}-\mathrm{Fe}$ and $\mathrm{Pt}-\mathrm{Co}$ interfaces, and smaller for $\mathrm{Au}-\mathrm{Co}$ and $\mathrm{Cu}-\mathrm{Co}$ interfaces. This regression analysis of experimental data with respect to a model that includes an interface does not of course prove, rather it strongly suggests the presence of interfaces.
The intrinsic magneto-optic figures of merit for $\mathrm{Pt}-\mathrm{Fe}$ and $\mathrm{Pt}-\mathrm{Co}$ are much larger than those for $\mathrm{Au}-\mathrm{Co}$ and $\mathrm{Cu}-$ Co. The largest figure of merit is for the Pt-Co system, and remains generally high throughout the spectral range. That is, there is seen to be little significant enhancement at shorter wavelengths, even in the Pt-Co system. This may occur because the figure of merit depends on a combination of both the magnetic (MO) response and the optical response. This could, for example, be manifested in a large Kerr rotation but low reflectance.

\section{ACKNOWLEDGMENTS}

Research was supported by the National Science Foundation, under Grant No. DMR-9623992, and by the Nebraska Research Initiative.

${ }^{1}$ P. F. Carcia, A. D. Meinhaldt, and A. Suna, Appl. Phys. Lett. 47, 178 (1985).

${ }^{2}$ H. J. G. Draaisma, W. J. M. De Jonge, and F. J. A. Den Broeder, J. Magn. Magn. Mater. 66, 351 (1987).

${ }^{3}$ P. F. Carcia, J. Appl. Phys. 63, 5066 (1988).

${ }^{4}$ W. B. Zeper, F. J. A. M. Greidamus, P. F. Carcia, and C. R. Fincher, J. Appl. Phys. 65, 4971 (1989).

${ }^{5}$ S. Hashimoto, Y. Ochiai, and K. Aso, Jpn. J. Appl. Phys. 28, L1824 (1989); J. Appl. Phys. 67, 2136 (1990).

${ }^{6}$ S. Hashimoto and Y. Ochiai, J. Magn. Magn. Mater. 88, 211 (1990).

${ }^{7}$ D. Weller, W. Reim, K. Sporl, and H. Brandle, J. Magn. Magn. Mater. 93, 183 (1991).

${ }^{8}$ K. Sato, H. Hongu, H. Ikekame, J. Watanabe, K. Tsuzukiyama, Y. Yogami, M. Fujisawa, and T. Fukazawa, Jpn. J. Appl. Phys. 31, 3603 (1992).

${ }^{9}$ K. Sato, in Magnet-Optical Spectra in Multilayers, edited by L. H. Bennett and R. E. Watson (World Scientific, Singapore, 1994), p. 277.

${ }^{10}$ M. T. Johnson, R. Jungblut, P. J. Kelly, and F. J. A. den Broeder, J. Magn. Magn. Mater. 148, 118 (1995).

${ }^{11}$ R. H. Victora and J. M. Maclaren, J. Appl. Phys. 73, 6415 (1993).

${ }^{12}$ D. Weller, R. F. C. Farrow, R. F. Marks, G. R. Harp, H. Notarys, and G. Gorman, Mater. Res. Soc. Symp. Proc. 313, 791 (1993).

${ }^{13}$ S. Uba, L. Uba, A. N. Yaresko, A. Y. Perlov, V. N. Antonov, and R. Gontarz, Phys. Rev. B 53, 6526 (1996).

${ }^{14}$ K. Sato, H. Ikekame, Y. Tosaka, K. Tsuzukiyama, Y. Togami, and M. Fujisawa, J. Magn. Magn. Mater. 126, 572 (1993).

${ }^{15}$ R. Atkinson, S. Pahirathan, I. W. Salter, P. J. Grundy, C. J. Tatnall, J. C. Lodder, and Q. Meng, J. Magn. Magn. Mater. 162, 131 (1996).

${ }^{16}$ N. K. Flevaris, S. Logothetidis, J. Petalas, P. Kielar, M. Nyvlt, V. Parizek, S. Visnovsky, and R. Krishnan, J. Magn. Magn. Mater. 121, 479 (1993).

${ }^{17}$ X. Gao, S. Heckens, and J. A. Woollam, J. Vac. Sci. Technol. A 16, 429 (1998).

${ }^{18}$ X. Gao, M. J. DeVries, D. W. Thompson, and J. A. Woollam, J. Appl. Phys. 83, 6747 (1998).

${ }^{19}$ X. Gao, D. W. Glenn, S. Heckens, D. W. Thompson, and J. A. Woollam, J. Appl. Phys. 82, 4525 (1997).

${ }^{20}$ C. M. Herzinger, B. Johs, W. A. McGahan, J. A. Woollam, and W. Paulson, J. Appl. Phys. 83, 3323 (1998).

${ }^{21}$ C. S. Wang and J. Callaway, Phys. Rev. B 9, 4897 (1974).

${ }^{22}$ P. M. Oppeneer and V. N. Antonov, in Spin-Orbit-Influeced Spectroscopy of Magnetic Solids, edited by H. Ebert and G. Schutz (Springer, Berlin, 1996), p. 29.

${ }^{23}$ H. Fu, Z. Yan, S. K. Lee, and M. Mansuripur, J. Appl. Phys. 78, 4076 (1995). 